\title{
Hybridization of stochastic reduced basis methods with polynomial chaos expansions
}

\author{
Sachin K. Sachdeva, Prasanth B. Nair *, Andy J. Keane \\ Computational Engineering and Design Group, University of Southampton, Southampton, SO17 1BJ, UK \\ Received 3 December 2004; received in revised form 14 August 2005; accepted 27 September 2005 \\ Available online 28 November 2005
}

\begin{abstract}
We propose a hybrid formulation combining stochastic reduced basis methods with polynomial chaos expansions for solving linear random algebraic equations arising from discretization of stochastic partial differential equations. Our objective is to generalize stochastic reduced basis projection schemes to non-Gaussian uncertainty models and simplify the implementation of higher-order approximations. We employ basis vectors spanning the preconditioned stochastic Krylov subspace to represent the solution process. In the present formulation, the polynomial chaos decomposition technique is used to represent the stochastic basis vectors in terms 'of multidimensional Hermite polynomials. The Galerkin projection scheme is then employed to compute the undetermined coefficients in the reduced basis approximation. We present numerical studies on a linear structural problem where the Youngs modulus is represented using Gaussian as well as lognormal models to illustrate the performance of the hybrid stochastic reduced basis projection scheme. Comparison studies with the spectral stochastic finite element method suggest that the proposed hybrid formulation gives results of comparable accuracy at a lower computational cost.
\end{abstract}

(C) 2005 Elsevier Ltd. All rights reserved.

Keywords: Stochastic finite element analysis; Projection schemes; Polynomial chaos; Stochastic reduced basis methods

\section{Introduction}

In recent years, there has been a resurgence of interest in developing numerical algorithms for analysis of systems governed by stochastic partial differential equations (PDEs). Such problems arise in a number of areas, including structural mechanics, fluid mechanics, heat transfer and flow through random porous media. The basic idea of uncertainty quantification is to estimate distributions for the field variables and subsequently for the outputs of interest, given probabilistic models for the PDE coefficients. In principle, the Monte Carlo simulation (MCS) technique can be readily applied to solve such problems to an arbitrary degree of accuracy, provided sufficient number of samples are used [1-3]. Even though significant advances have been made in improving the efficiency of the MCS technique, the associated computational cost can be prohibitive for problems with large number of degrees of freedom (DOF).

\footnotetext{
* Corresponding author.

E-mail address: p.b.nair@soton.ac.uk (P.B. Nair).

URL: http://www.soton.ac.uk/ pbn.
}

0266-8920/\$ - see front matter (C) 2005 Elsevier Ltd. All rights reserved. doi:10.1016/j.probengmech.2005.09.003
Perturbation and sensitivity based methods offer computationally efficient alternatives to the MCS technique and have been popularly applied to compute the first two statistical moments of the response quantities; see, for example, [4-9]. However, the major drawback of such local approximation techniques is that the results become inaccurate when the coefficients of variation of the input random variables are increased.

An alternative approach referred to as the spectral stochastic finite element method (SSFEM) was pioneered by Ghanem and Spanos [10]. The SSFEM is based on a spectral representation of uncertainty in terms of multi-dimensional Hermite polynomials also referred to as polynomial chaos (PC) expansions. The solution process is treated as an element in the Hilbert space of random functions and is approximated by its projection onto a finite subspace spanned by orthogonal Hermite polynomials. A Galerkin projection scheme is used to convert the original stochastic PDE into a set of coupled deterministic PDEs. In recent years, the spectral approach has been applied to solve a wide class of stochastic problems namely, elasticity problems [10], random vibration [11], soil mechanics [12], transport process in heterogeneous media [13], plasticity problems [14], fluid dynamics [15], mid-frequency structural dynamics [16], and wave propagation in random media [17]. It has been shown that unlike perturbation-based 
simplicity. However, in some cases, it may be preferable to use a lognormal process to represent uncertainty, particularly when the quantity under consideration is constrained to be always positive. This is true when modeling the Youngs modulus, conductivity or permeability of a random heterogeneous solid. A lognormal process can be written as the exponential of a Gaussian process as follows [43]

$h_{l}(\mathbf{x} ; \omega)=\exp (h(\mathbf{x} ; \omega))$.

Discretizing the random field $h(\mathbf{x} ; \omega)$ as earlier using the $\mathrm{KL}$ expansion scheme and substituting in the preceding equation gives

$\hat{h}_{l}(\mathbf{x} ; \omega)=\exp \left(\langle h(\mathbf{x} ; \omega)\rangle+\sum_{i=1}^{N} \sqrt{\lambda_{i}} \theta_{i} h_{i}(x)\right)$.

Since $\hat{h}_{l}(\mathbf{x} ; \omega)$ is a random function it can be decomposed using the $\mathrm{PC}$ expansion scheme as follows

$\hat{h}_{l}(\mathbf{x} ; \omega)=\sum_{i=0}^{P_{1}} h_{i}^{l} \Gamma_{i}(\boldsymbol{\theta})$

where $\Gamma_{i}(\theta)$ are multidimensional Hermite polynomials in $\theta_{1}, \theta_{2}, \ldots, \theta_{N}$ and $h_{i}^{l}$ are the coefficients of expansion.

It follows from Eqs. (14) and (9) that the global stiffness matrix for a lognormal model can be written in a form similar to the Gaussian case, i.e.

$K(\theta)=\sum_{i=0}^{P_{1}} \mathbf{K}_{i}^{l} \Gamma_{i}(\theta)$.

A Gaussian stochastic process can be considered as a special case of lognormal stochastic processes since we recover Eq. (11) when only the first order terms in the PC expansion are retained in Eq. (15). We would also point out that even though the discussion in this section was focused on a structural problem, the semi-discretized form of the governing equations are similar to those obtained when modeling steady-state heat transfer [18] or fluid flow in a random heterogeneous media [36].

\section{Stochastic Krylov subspace}

Let us next consider in more detail the system of linear random algebraic equations of the form $\mathbf{K}(\boldsymbol{\theta}) \mathbf{u}(\boldsymbol{\theta})=\mathbf{f}(\boldsymbol{\theta})$, where the coefficient matrix $\mathbf{K}(\theta)$ is given by Eq. (15). This equation can be rewritten in a more convenient form as

$$
\left(\mathbf{K}_{0}+\sum_{i=1}^{P_{1}} \mathbf{K}_{i} \Gamma_{i}(\boldsymbol{\theta})\right) \mathbf{u}(\theta)=\mathbf{f}
$$

where $\mathbf{K}_{0}$ is the mean stiffness matrix and $\mathbf{K}_{i}$ are weighted stiffness matrices. $P_{1}$ depends on the order of $\mathrm{KL}$ expansion used to discretize the field for the case of Gaussian stochastic process; see Eq. (8). For lognormal stochastic processes, $P_{1}$ depends on the order of KL expansion as well as the order of the PC decomposition used in Eq. (14).

Stochastic reduced basis methods [26,27] approximate the solution of Eq. (16) using basis vectors spanning the stochastic
Krylov subspace defined below

$\mathcal{K}_{m}(\mathbf{K}(\boldsymbol{\theta}), \mathbf{f})=\operatorname{span}\left\{\mathbf{f}, \mathbf{K}(\boldsymbol{\theta}) \mathbf{f}, \mathbf{K}(\boldsymbol{\theta})^{2} \mathbf{f}, \cdots ; \mathbf{K}(\boldsymbol{\theta})^{m-1} \mathbf{f}\right\}$.

The following theorem motivates the application of the stochastic Krylov subspace for solving Eq. (10) [27].

Theorem 1: If the minimal random polynomial of a nonsingular random square matrix $\mathbf{K}(\theta)$ has degree $m$, then the solution to $\mathbf{K}(\theta) \mathbf{u}(\theta)=\mathbf{f}$ lies in the stochastic Krylov subspace $\mathcal{K}_{m}(\mathbf{K}(\theta), \mathbf{f})$.Hence, a stochastic reduced basis approximation of the solution process can be written as

$\hat{\mathbf{u}}(\boldsymbol{\theta})=\xi_{0} \psi_{0}(\boldsymbol{\theta})+\xi_{1} \psi_{1}(\boldsymbol{\theta})+\cdots+\xi_{n} \psi_{m}(\boldsymbol{\theta})=\Psi(\boldsymbol{\theta}) \xi$,

where $\boldsymbol{\Psi}(\boldsymbol{\theta})=\left\{\psi_{0}(\boldsymbol{\theta}), \psi_{1}(\boldsymbol{\theta}), \ldots, \psi_{m}(\boldsymbol{\theta})\right\} \in \mathbb{R}^{n \times(m+1)}$ is a set of basis vectors spanning the stochastic Krylov subspace $\mathcal{K}_{m}(\mathbf{K}($ $\theta), \mathbf{f}))$ and $\xi=\left\{\xi_{0}, \xi_{1}, \ldots, \xi_{m}\right\}^{\mathrm{T}} \in \mathbb{R}^{m+1}$ is a vector of undetermined coefficients.

It is to be noted here that the number of basis vectors required to compute accurate approximations depends on the degree of overlap of the PDFs of the eigenvalues of the coefficient matrix $\mathbf{K}(\theta)$ [27]. To ensure good approximations using a small number of basis vectors, it is preferable to use a preconditioner. The key idea here is to transform the coefficient matrix such that the PDFs of the modified eigenvalues numerically tend to have a high degree of overlap. Following $[26,27]$, we use the deterministic matrix $\langle\mathbf{K}(\theta)\rangle^{-1}=\mathbf{K}_{0}^{-1}$ as the pre-conditioner. This choice is motivated by the observation that $\mathbf{K}_{0}^{-1} \mathbf{K}(\theta)$ will numerically behave like a matrix with a small number of distinct eigenvalues, particularly for small randomness. Note here that, in theory, convergence can be guaranteed as long as the preconditioner is invertible. However, by using the preconditioner suggested here, convergence can be significantly accelerated - in other words, it becomes possible to achieve high accuracy using a small number of basis vectors.

The first three basis vectors spanning the preconditioned stochastic Krylov subspace can be written as

$$
\begin{aligned}
\psi_{0}(\boldsymbol{\theta}) & =\mathbf{K}_{0}^{-1} \mathbf{f}, \quad \psi_{1}(\boldsymbol{\theta})=\mathbf{K}_{0}^{-1} \mathbf{K}(\boldsymbol{\theta}) \psi_{0}(\boldsymbol{\theta}) \text { and } \psi_{2}(\boldsymbol{\theta}) \\
& =\mathbf{K}_{0}^{-1} \mathbf{K}(\boldsymbol{\theta}) \psi_{1}(\boldsymbol{\theta}) .
\end{aligned}
$$
For Gaussian random fields when $\mathbf{K}(\theta)=\mathbf{K}_{0}+\sum_{i=1}^{N} \theta_{i} \mathbf{K}_{i}$, the
basis vectors simplify to the form

$$
\begin{aligned}
\psi_{0}(\boldsymbol{\theta}) & =\mathbf{u}_{0}, \psi_{1}(\boldsymbol{\theta})=\sum_{i=1}^{N} \mathbf{d}_{i} \boldsymbol{\theta}_{i} \text { and } \psi_{2}(\boldsymbol{\theta}) \\
& =\sum_{i=1}^{N} \sum_{j=1}^{N} \mathbf{e}_{i j} \boldsymbol{\theta}_{i} \boldsymbol{\theta}_{j},
\end{aligned}
$$

where $\quad \mathbf{u}_{0}=\mathbf{K}_{0}^{-1} \mathbf{f} \in \mathbb{R}^{n}, \quad \mathbf{d}_{i}=\mathbf{K}_{0}^{-1} \mathbf{K}_{i} \mathbf{u}_{0} \in \mathbb{R}^{n} \quad$ and $\mathbf{e}_{i j}=\mathbf{K}_{0}^{-1} \mathbf{K}_{i} \mathbf{d}_{j} \in \mathbb{R}^{n}$.

Nair and Keane [27] and Sachdeva et al. [29] have applied reduced basis methods using upto three basis vectors for static and dynamic analysis of structural systems. Using the representation given in Eqs. (19) and (20) it is cumbersome to implement higher order projection schemes; for example 
the fourth and fifth basis vectors would look like

$$
\begin{aligned}
& \psi_{3}(\boldsymbol{\theta})=\sum_{k=1}^{N} \mathbf{f}_{i j k} \theta_{i} \theta_{j} \theta \text { and } \\
& \psi_{4}(\boldsymbol{\theta})=\sum_{i=1}^{N} \sum_{j=1}^{N} \sum_{k=1}^{N} \sum_{l=1}^{N} \mathbf{g}_{i j k l} \theta_{i} \theta_{j} \theta_{k} \theta_{l},
\end{aligned}
$$

where $\mathbf{f}_{i j k}=\mathbf{K}_{0}^{-1} \mathbf{K}_{i} \mathbf{e}_{j k} \in \mathbb{R}^{n}$ and $\mathbf{g}_{i j k l}=\mathbf{K}_{0}^{-1} \mathbf{K}_{i} \mathbf{f}_{j k l} \in \mathbb{R}^{n}$.

It is to be noted here that the basis vectors in Eqs. (20) and (21) only apply to the case when the random field is Gaussian. Hence, an alternative representation is required for nonGaussian uncertainty models. It follows from Eq. (19) that the $k$ th basis vector can be written as $\psi_{k}=\left(\mathbf{K}_{0}^{-1} \mathbf{K}(\boldsymbol{\theta})\right)^{k} \mathbf{K}_{0}^{-1} \mathbf{f}$. Since the basis vectors are functions of random variables, it is proposed to apply PC expansions to arrive at a numerically more tractable description. Further, each basis vector can be computed recursively as $\psi_{k+1}=\mathbf{K}_{0}^{-1} \mathbf{K}(\theta) \psi_{k}$. Hence, by making use of the idea of pseudo-spectral expansions [30] and the recursive representation of basis vectors, it becomes possible to write all the higher order basis vectors in terms of multidimensional Hermite polynomials.

The first basis vector $\psi_{1}(\theta)=\mathbf{K}_{0}^{-1} \mathbf{f}$ can be rewritten in the following form

$\hat{\psi}_{1}(\theta)=\sum_{i=0}^{P_{2}} \psi_{i}^{1} \Gamma_{i}$,

where $P_{2}$ depends on the order of $\mathrm{PC}$ expansion used for decomposing the basis vectors and

$\psi_{i}^{1}=\left\{\begin{array}{cc}\mathbf{K}_{0}^{-1} \mathrm{f} & \forall i=0 \\ 0 & \forall 1 \leq i \leq P_{2}\end{array}\right.$

Since higher order basis vectors need more terms for better approximation, we shall use PC expansions of appropriate order to minimize truncation errors. Further, since we make use of the recursive representation of the basis vectors, each basis vector should be computed using a PC expansion one order higher than the preceding one. Here, for simplicity of notation we assume $P_{2}$ as the number of terms used to approximate all the basis vectors (clearly, $P_{2}$ should be sufficiently high to approximate the highest order basis vector accurately).

Next, the PC expansion of the second basis vector $\psi_{2}(\theta)=$ $\mathbf{K}_{0}^{-1} \mathbf{K}(\theta) \psi_{1}(\theta)$ can be written as

$\hat{\psi}_{2}=\sum_{i=0}^{P_{2}} \psi_{i}^{2} \Gamma_{i}$

where $\psi_{i}^{2}=\mathbf{K}_{0}^{-1} \mathbf{K}_{i} u_{0} \in \mathbb{R}^{n}$.

Now, taking advantage of the recursive representation of the stochastic basis vectors, the higher order basis vectors can readily be written as functions of random variables. The third basis vector $\psi_{3}(\theta)=\mathbf{K}_{0}^{-1} \mathbf{K}(\theta) \psi_{2}(\theta)$ can be written as follows by making use of Eqs. (16) and (24)

$$
\hat{\psi}_{3}(\theta)=\mathbf{K}_{0}^{-1} \sum_{i=0}^{P_{1}} \sum_{j=0}^{P_{2}} \mathbf{K}_{i} \psi_{j}^{2} \Gamma_{i} \Gamma_{j} .
$$

Note that the above equation contains the product of two Hermite polynomials. For a more tractable computational treatment, each basis vector should be represented as a linear combination of a deterministic vector and a Hermite polynomial. We eventually want to represent the third and all higher order basis vectors in the form

$\hat{\psi}_{3}(\theta)=\sum_{i=0}^{P_{2}} \psi_{i}^{3} \Gamma_{i}$.

Equating the right hand sides of Eqs. (25) and (26) and multiplying both sides by $\Gamma_{k}$ gives the following

$\sum_{i=0}^{P_{2}} \psi_{i}^{3} \Gamma_{i} \Gamma_{k}=\mathbf{K}_{0}^{-1} \sum_{i=0}^{P_{2}} \sum_{i=0}^{P_{2}} \mathbf{K}_{i} \psi_{j}^{2} \Gamma_{i} \Gamma_{j} \Gamma_{k}$.

Applying the expectation operator $\langle\cdot\rangle$ on the previous equation and using the orthogonality property of the PC basis gives the following expression for the deterministic vector $\psi_{k}^{3}$ $\in \mathbb{R}^{n}$

$\psi_{\mathbf{k}}^{3}=\frac{\mathbf{K}_{0}^{-1} \sum_{i=0}^{P_{2}} \sum_{j=0}^{P_{2}} \mathbf{K}_{i} \psi_{j}^{2} \mathbf{D}_{i j k}}{\left\langle\Gamma_{k}^{2}\right\rangle}$

where the tensor $\mathbf{D}_{i j k}=\left\langle\Gamma_{i} \Gamma_{j} \Gamma_{k}\right\rangle$. We can compute all other basis vectors on similar lines. To make the computations efficient the tensor $\mathbf{D}_{i j k}$ can be calculated beforehand during the preprocessing stage. It is worth noting that in the approach presented here, we are using a pseudo-spectral approach [30] to compute the higher-order basis vectors to avoid the computational effort and memory requirement associated with calculation and storage of high-order tensors of the form $\left\langle\Gamma_{i_{1}}\right.$ $\left.\Gamma_{i_{2}} \cdots \Gamma_{i_{p}}\right\rangle$ (these higher-order tensors would arise if the PC expansion scheme is directly applied to basis vectors represented in the original form $\left.\psi_{k}=\left[\mathbf{K}_{0}^{-1} \mathbf{K}(\theta)\right]^{k} \mathbf{K}_{0}^{-1} \mathbf{f}\right)$.

A general expression for the deterministic vector $\psi_{k}^{m+1}$ (for $m>1$ ) can be written in terms of the third-order tensor $\mathbf{D}_{i j k}$ as

$\psi_{k}^{m+1}=\frac{\mathbf{K}_{0}^{-1} \sum_{i=0}^{P_{2}} \sum_{j=0}^{P_{2}} \mathbf{K}_{i} \psi_{j}^{m} \mathbf{D}_{i j k}}{\left\langle\Gamma_{k}^{2}\right\rangle}$

The general expression for the stochastic basis vector $\hat{\psi}_{m+1}$ $(\theta)$ can then be written as

$\hat{\psi}_{m+1}(\theta)=\sum_{i=1}^{P_{2}} \psi_{i}^{m+1} \Gamma_{i}$

Finally, the set of stochastic basis vectors can be written as $\hat{\Psi}(\theta)=\left[\hat{\psi}_{0}(\theta), \hat{\psi}_{1}(\theta) \ldots, \hat{\psi}_{m}(\theta)\right]$. It is to be noted here that these reformulated basis vectors are not exactly the same as those defined earlier in Eq. (17), because of the PC decomposition. However, if PC expansions of appropriate order are used, the basis vectors would span the preconditioned stochastic Krylov subspace more closely. It can also be noted from the derivation 
that the computational complexity involved in constructing the PC expansion of the basis vectors will grow when the number of random variables is increased. This observation follows from Eqs. (29) and (30) in which the number of matrix-vector operations required to evaluate the PC expansion coefficients $\psi_{k}^{m+1}$ is a function of $P_{2}$ which in turn depends on the total number of random variables.

As in Eq. (18) the stochastic reduced basis representation for the response process can be written as

$\hat{\mathbf{u}}(\boldsymbol{\theta})=\hat{\xi}_{0} \hat{\psi}_{0}(\boldsymbol{\theta})+\hat{\xi}_{1} \hat{\psi}_{1}(\boldsymbol{\theta})+\cdots+\hat{\xi}_{n} \hat{\psi}_{m}(\boldsymbol{\theta})=\hat{\boldsymbol{\Psi}}(\boldsymbol{\theta}) \hat{\xi}$,

where $\hat{\xi}=\left\{\hat{\xi}_{0}, \hat{\xi}_{1}, \ldots, \hat{\xi}_{m}\right\}^{\mathrm{T}} \in \mathbb{R}^{m+1}$ is a vector of undetermined coefficients.

\section{Stochastic projection scheme}

Substituting Eq. (31) into the governing Eq. (16), we arrive at the following system of linear random algebraic equations

$$
\left(\sum_{i=0}^{P_{1}} \mathbf{K}_{i} \Gamma_{i}\right) \hat{\boldsymbol{\Psi}}(\boldsymbol{\theta}) \hat{\xi}=\mathbf{f}
$$

A projection scheme needs to be applied to the above system of equations to estimate the undetermined coefficients $\hat{\xi}$. An orthogonal Galerkin projection scheme is appropriate when $\mathbf{K}(\theta)$ is a symmetric positive definite matrix. We present next a Galerkin projection scheme in which orthogonality is imposed in the sense of Eq. (2).

\subsection{Galerkin scheme}

The stochastic residual error vector can be written as

$\varepsilon(\boldsymbol{\theta})=\left(\sum_{i=0}^{P_{1}} \mathbf{K}_{i} \Gamma_{i}\right) \hat{\boldsymbol{\Psi}}(\boldsymbol{\theta}) \hat{\xi}-\mathbf{f}$.

In the Galerkin projection scheme, the residual vector $\varepsilon(\theta)$ is made orthogonal to all the stochastic basis vectors, i.e.

$$
\left(\sum_{i=0}^{P_{1}} \mathbf{K}_{i} \Gamma_{i}\right) \hat{\boldsymbol{\Psi}}(\boldsymbol{\theta}) \hat{\xi}-\mathbf{f} \perp \hat{\psi}_{i}(\boldsymbol{\theta}), \quad \forall j=0,1,2, \ldots, m .
$$

By imposing the above orthogonality condition in the sense of Eq. (2), we obtain a reduced-order system of $(m+1) \times(m+$ 1) deterministic linear algebraic equations of the form

$$
\left\langle\sum_{i=0}^{p_{1}} \Gamma_{i} \hat{\Psi}(\boldsymbol{\theta})^{\mathrm{T}} \boldsymbol{K}_{i} \hat{\Psi}(\boldsymbol{\theta}) \hat{\xi}=\left\langle\hat{\Psi}(\boldsymbol{\theta})^{\mathrm{T}} \mathbf{f}\right\rangle\right.
$$

Without any loss of generality the stochastic reduced basis approximation in Eq. (31) can be rearranged and rewritten as follows

$\hat{\mathbf{u}}(\boldsymbol{\theta})=\left[\sum_{i=0}^{P_{2}} \Pi_{i} \Gamma_{i}\right] \hat{\boldsymbol{\xi}}$,

where $\Pi_{i}=\left[\psi_{i}^{0}, \psi_{i}^{1}, \ldots, \psi_{i}^{m}\right] \in \mathbb{R}^{n \times(m+1)}$ is a deterministic matrix.
Substituting Eq. (36) into Eq. (35) gives the following system of equations

$$
\begin{aligned}
& \left\langle\left[\sum_{i=0}^{P_{2}} \Pi_{i}^{\mathrm{T}} \Gamma_{i}\right]\left[\sum_{j=0}^{P_{1}} \boldsymbol{K}_{j} \Gamma_{j}\right]\left[\sum_{k=0}^{P_{2}} \Pi_{k} \Gamma_{k}\right]\right\rangle \hat{\boldsymbol{\xi}} \\
& =\left\langle\left[\sum_{i=0}^{P_{2}} \Pi_{i}^{T} \Gamma_{i}\right] \mathbf{f}\right\rangle .
\end{aligned}
$$

Simplifying the preceding equation gives

$$
\left(\sum_{i=0}^{P_{2}} \sum_{j=0}^{P 1} \sum_{k=0}^{P_{2}} \Pi_{i}^{\mathrm{T}} \mathbf{K}_{j} \Pi_{k}\left\langle\Gamma_{i} \Gamma_{j} \Gamma_{k}\right\rangle\right) \xi=\sum_{i=0}^{P_{2}} \Pi_{i}^{\mathrm{T}} \mathbf{f}\left\langle\Gamma_{i}\right\rangle .
$$

Substituting $\mathbf{D}_{i j k}=\left\langle\Gamma_{i} \Gamma_{j} \Gamma k\right\rangle$ and using the fact that $\left\langle\Gamma_{i}\right\rangle=0$ for $i>0$ and $\Gamma_{0}=1$, the above equation can be compactly written as

$$
\left(\sum_{i=0}^{P_{2}} \sum_{j=0}^{P_{1}} \sum_{k=0}^{P_{2}} \Pi_{i}^{\mathrm{T}} \mathbf{K}_{j} \Pi_{k} \mathbf{D}_{i j k}\right) \hat{\boldsymbol{\xi}}=\Pi_{0}^{\mathrm{T}} \mathbf{f}
$$

Eq. (39) is a reduced order deterministic system of linear equations, which can be solved for the coefficient vector $\hat{\xi}$. These coefficients can then be used in conjunction with Eq. (31) to approximate the solution process $\hat{\mathbf{u}}(\boldsymbol{\theta})$.

\subsection{Connections between SRBMS and SSFEM}

In the SSFEM of Ghanem and Spanos [10], the solution process is approximated using a PC expansion scheme as follows

$\hat{\mathbf{u}}(\theta)=\sum_{i=0}^{P_{2}} \mathbf{u}_{i} \Gamma_{i}(\theta)$

where $\mathbf{u}_{i} \in \mathbb{R}^{n}$ are vectors of undetermined coefficients and $P_{2}$ depends on the order of PC expansion used for the solution process. In contrast to SRBMs which have only $m+1$ undetermined coefficients, the SSFEM postulates an approximation withn $n\left(P_{2}+1\right)$ unknowns. It can be clearly seen from (36) and (40) that if we set $\mathbf{u}_{i}=\Pi_{i} \hat{\xi}$, the final form of the solution process is the same for the hybrid scheme and the SSFEM.

In the SSFEM, the PC expansion in (40) is substituted into the governing Eq. (16) and the Galerkin scheme is applied, which leads to the following deterministic system of linear algebraic equations

$\sum_{i=0}^{P_{1}} \sum_{j=0}^{P_{2}} \boldsymbol{K}_{i} \boldsymbol{u}_{j}\left\langle\Gamma_{i} \Gamma_{j} \Gamma_{k}\right\rangle=\left\langle\Gamma_{k} f\right\rangle, k=0,1,2, \ldots, P_{2}$.

It is interesting to note that the equation for the undetermined coefficients in (39) obtained using SRBMs is quite similar to that obtained using the SSFEM. Despite the fact that eqs. (39) and (41) look similar they give rise to linear algebraic equations with very different dimensionalities. Specifically, the SSFEM approach leads to a system of equations with much increased dimensionality $-n\left(P_{2}+1\right) \times$ 
$n\left(P_{2}+1\right)$. In practice, $P_{2}$ can be $\mathcal{O}(10)$ for problems with modest number of random variables. Hence, the application of the SSFEM to problems with large number of DOF and random variables may become computationally prohibitive. In contrast, SRBMs lead to a reduced-order $(m+1) \times(m+1)$ system of equations, where the number of basis vectors typically required in practice to ensure good approximations is around three or four.

\section{Post-processing}

Since the final expression for the solution process is an expansion in PC basis functions, it is straightforward to compute various statistics in the post-processing stage. In this section we derive expressions for the statistical moments of the solution and the norm of the stochastic residual error.

\subsection{Statistical moments}

Applying the expectation operator to Eq. (36) gives the mean of the response process as

$\langle\hat{u}\rangle=\left\langle\left[\sum_{i=0}^{P_{2}} \Pi_{i} \Gamma_{i}\right]\right\rangle \hat{\xi}$

which reduces to the following simple expression due to the properties of the $\mathrm{PC}$ basis functions.

$\langle\hat{\mathbf{u}}\rangle=\Pi_{0} \hat{\xi}$.

The covariance matrix of the response process can be computed as

$\mathbf{u}_{\mathrm{Cov}}=\left\langle\left[(\hat{\mathbf{u}}(\boldsymbol{\theta})-\langle\hat{\mathbf{u}}\rangle)(\hat{\mathbf{u}}(\theta)-\langle\hat{\mathbf{u}}\rangle)^{\mathrm{T}}\right]\right.$

Substituting Eqs. (36) and (43) into Eq. (44) gives the following expression for the response covariance matrix

$$
\begin{aligned}
\mathbf{u}_{\text {Cov }} & =\sum_{i=0}^{P_{2}} \sum_{j=0}^{P_{2}}\left[\Pi_{i} \xi \xi^{\mathrm{T}} \Pi_{j}^{\mathrm{T}}\right]\left\langle\Gamma_{i} \Gamma_{j}\right\rangle-\Pi_{0} \xi \xi^{\mathrm{T}} \sum_{i=0}^{P_{2}} \Pi_{i}^{\mathrm{T}}\left\langle\Gamma_{i}\right\rangle \\
& -\sum_{i=0}^{P_{2}} \Pi_{i}\left\langle\Gamma_{i}\right\rangle \xi \xi^{\mathrm{T}} \Pi_{0}+\Pi_{0} \xi \xi^{\mathrm{T}} \Pi_{0}^{\mathrm{T}}
\end{aligned}
$$

which simplifies to the following after some algebraic manipulations

$\mathbf{u}_{C o \nu}=\sum_{i=0}^{P_{2}}\left[\Pi_{i} \xi \xi^{\mathrm{T}} \Pi_{i}^{\mathrm{T}}\right]\left\langle\Gamma_{i}^{2}\right\rangle-\Pi_{0} \xi \xi^{\mathrm{T}} \Pi_{0}^{\mathrm{T}}$.

Since the hybrid projection scheme leads to an explicit expression for the solution process, its complete statistical characterization becomes computationally feasible. For example, we can employ simulation schemes in conjunction with kernel density estimation techniques [44] to approximate the PDF of the response quantities given the final expression for the solution process in terms of a random polynomial. Alternatively, the wide body of numerical methods developed for reliability analysis such as the first-order reliability method
(FORM), second-order reliability method (SORM) and importance sampling can be applied to the explicit expressions for the solution process [39].

\subsection{Error norm of the residual}

We now focus on computing the norm of the residual error $\varepsilon(\theta)$. Since $\varepsilon(\theta)$ is a random function it can be decomposed using the $\mathrm{PC}$ expansion as follows

$\boldsymbol{\varepsilon}(\boldsymbol{\theta})=\left(\sum_{i=0}^{P_{1}} \mathbf{K}_{i} \Gamma_{i}\right)\left(\sum_{j=0}^{P_{2}} \Pi_{j} \boldsymbol{\Gamma}_{j}\right) \hat{\xi}-\mathbf{f}=\sum_{i=0}^{P_{3}} \varepsilon_{i} \Gamma_{i}$,

where $P_{3}$ is related to the order of $\mathrm{PC}$ decomposition used. Note that the order of the decomposition used here should be greater than the order used to decompose the stochastic basis vectors in order to minimize truncation errors, i.e. $P_{3}=P_{1}+P_{2}$.

Rearranging the preceding equation and multiplying both sides by $\Gamma_{k}$ gives

$$
\left[\left(\sum_{i=0}^{P_{1}} \sum_{j=1}^{P_{2}} \mathbf{K}_{i} \Pi_{j} \Gamma_{i} \Gamma_{j}\right) \hat{\boldsymbol{\xi}}-\mathbf{f}\right] \Gamma_{k}=\sum_{i=0}^{P_{3}} \varepsilon_{i} \Gamma_{i} \Gamma_{k} .
$$

Ensemble averaging of the above equation and using the properties of $\mathrm{PC}$ basis functions give the following expression for $\varepsilon_{k}$

$\varepsilon_{k}=\frac{\sum_{i=0}^{P_{1}} \sum_{j=0}^{P_{2}} \mathbf{K}_{i} \Pi_{j} \hat{\xi} \mathbf{D}_{i j k}-\mathbf{f}\left\langle\Gamma_{k}\right\rangle}{\left\langle\Gamma_{k}^{2}\right\rangle}$

Hence, the final expression for $\varepsilon(\theta)$ is

$\varepsilon(\theta)=\sum_{i=0}^{P_{3}}\left[\frac{\sum_{i=0}^{P_{1}} \sum_{j=0}^{P_{2}} \mathbf{K}_{i} \Pi_{j} \hat{\xi} \mathbf{D}_{i j k}-\mathbf{f}\left\langle\Gamma_{k}\right\rangle}{\left\langle\Gamma_{k}^{2}\right\rangle}\right] \Gamma_{l}$.

The $L_{2}$ norm of the residual error can hence be written as

$\|\varepsilon(\theta)\|=\left\langle\varepsilon(\theta)^{\mathrm{T}} \varepsilon(\theta)\right\rangle=\sum_{i=0}^{P_{2}} \sum_{j=0}^{P_{3}} \varepsilon_{i}^{\mathrm{T}} \varepsilon_{j}\left\langle\Gamma_{i} \Gamma_{j}\right\rangle$.

The $L_{2}$ norm of the error can be used to analyze the convergence trends of reduced basis methods of different orders. It can also be used to estimate the error in the solution when validation studies using MCS are too expensive [26].

\section{Numerical studies}

In this section we present numerical studies to illustrate the application of hybrid SRBMs to a model stochastic PDE in structural mechanics. The performance of the projection schemes depends on the number of basis vectors, the type of covariance function used to model uncertainty, the coefficient of variation of the input random field, the number of random variables arising from $\mathrm{KL}$ expansion of random fields and the number of terms retained in the PC expansions. We study 
the accuracy of the hybrid projection schemes as a function of the standard deviation of the input random field $(\sigma)$. Results are presented for both Gaussian as well as lognormal models. The order of PC expansion is chosen to be sufficiently high to minimize truncation errors due to pseudo-spectral decomposition of the stochastic basis vectors. The results are compared against MCS based on deterministic structural analysis. The $L_{2}$ norm of the residual is also computed to investigate the convergence trends of the hybrid projection scheme when the number of stochastic basis vectors is increased.

The problem considered here is a thin square plate of unit length (i.e. $[0,1] \times[0,1]$ ) clamped at one edge and subjected to uniform in-plane tension at the opposite edge (from [10]). This problem has been used earlier in the literature [39] for studying the convergence behavior of PC projection schemes - we use some of the functions from the SSFEM Matlab toolbox developed there in our numerical studies. The random field representing uncertainty in the Youngs modulus of this structure is discretized using the standard $\mathrm{KL}$ expansion procedure. The exponential correlation function shown below is used to represent uncertainty

$h(\mathbf{x}, \mathbf{y})=\exp \left(-\frac{\left|x_{1}-x_{2}\right|}{b_{1}}-\frac{\left|y_{1}-y_{2}\right|}{b_{2}}\right)$.

The domain of the plate is discretized into 16 square four noded quadrilateral elements as shown in Fig. 1, which leads to a total of 50 dof considering 2 dof per node. The external loads are assumed to be deterministic and of unit magnitude. The modulus of elasticity of the plate is modeled first as a twodimensional Gaussian random field with the exponential correlation model given in Eq. (53) with $b_{1}=b_{2}=1$. The random field is discretized using the $\mathrm{KL}$ expansion scheme and four terms are retained.

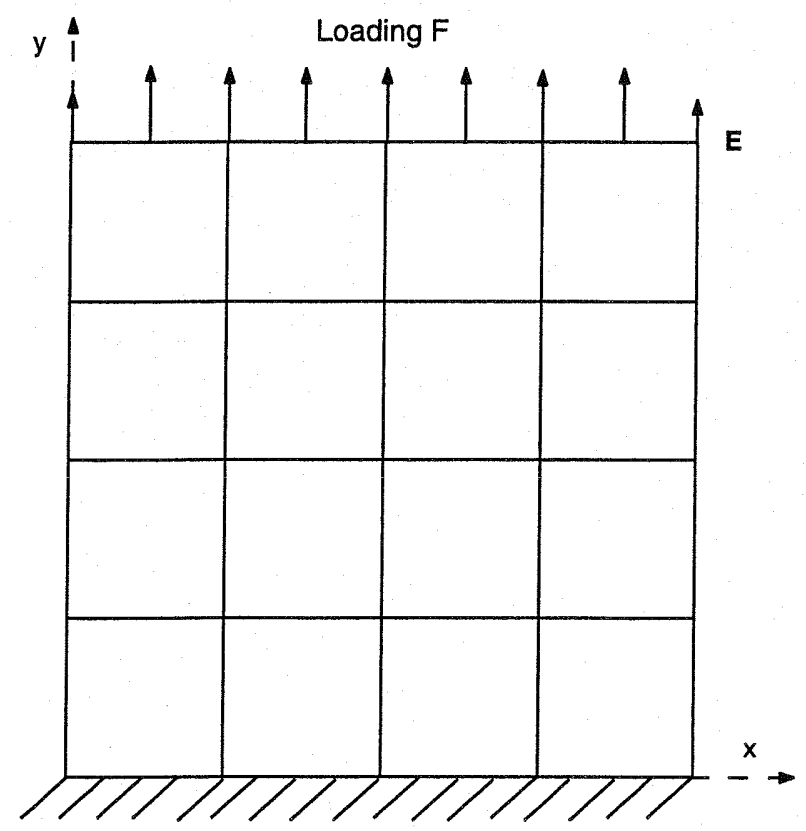

Fig. 1. Schematic of plate problem.

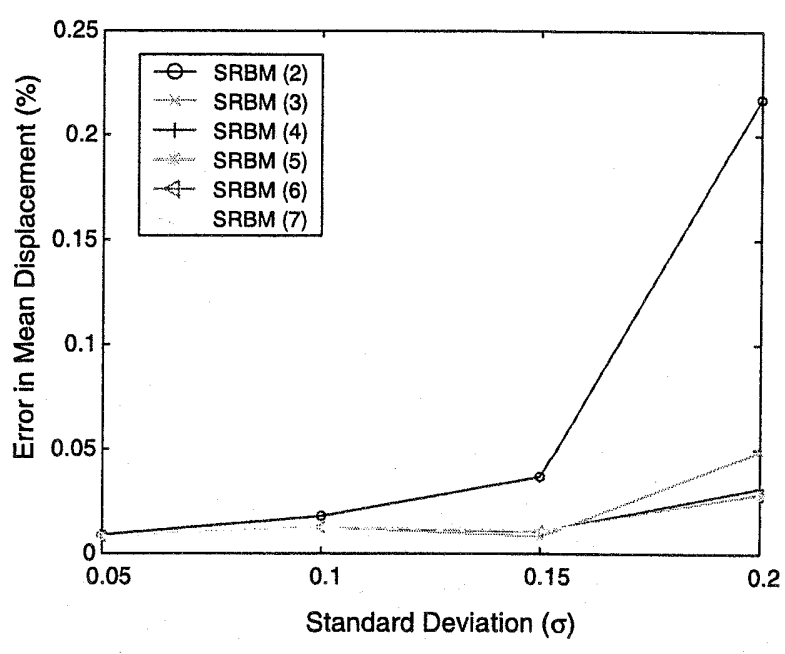

Fig. 2. Percentage error in the mean displacement at point ' $E$ ' using up to 7 basis vectors as a function of the standard deviation of the random Youngs modulus (Gaussian model).

For Gaussian random fields, SRBMs with up to seven stochastic basis vectors are applied to compute the first two statistical moments of the displacement. Response statistics are computed for $\sigma=0.05,0.10,0.15$ and 0.20 . For each case, MCS with a sample size of 500,000 is used to generate benchmark results against which all other results are compared.

The percentage errors in the mean and the standard deviation of the vertical displacement at the corner ' $E$ ' (see Fig. 1) for different values of $\sigma$ are shown in Figs. 2 and 3, respectively. Note that in the figs. we use SRBM $(m)$ to denote the case when ' $m$ ' stochastic basis vectors are used. For $\sigma<$ 0.15 , high quality approximations can be achieved using only three basis vectors. However, when $\sigma$ is increased further, around four-five basis vectors are required to ensure that the approximation errors are lower than 1\%. Fig. 4 shows the convergence of the $L_{2}$ norm of the error as a function of the number of basis vectors when $\sigma$ is increased. It can be observed

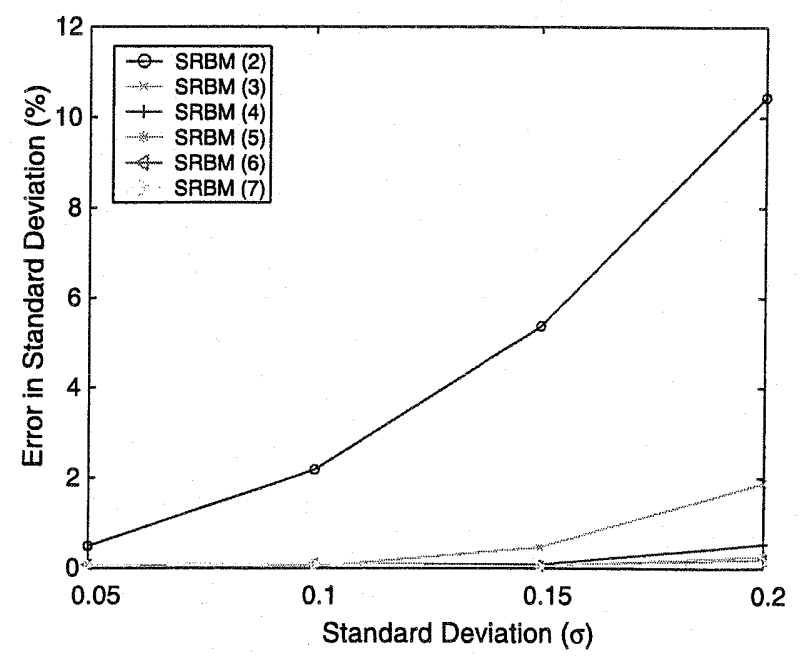

Fig. 3. Percentage error in the standard deviation of displacement at point ' $E$ ' using up to 7 basis vectors as a function of the standard deviation of the random Youngs modulus (Gaussian model). 


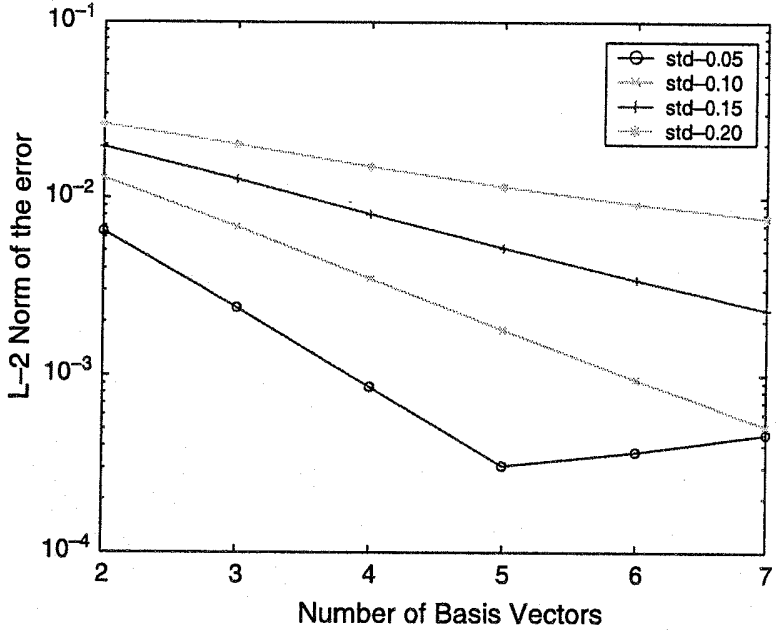

Fig. 4. Convergence of the $L_{2}$ norm of the residual error when the number of basis vectors is increased (Gaussian model).

from the results that the error is lower for lower standard deviation of the random field and it converges rapidly for all values of $\sigma$ as the number of basis vectors is increased.

We next consider the case when a lognormal model is used to represent uncertainty in the Youngs modulus. Here, we compute the mean and standard deviation of the displacement when $\sigma$ is increased up to 0.5 . The lognormal field is generated by exponentiating the underlying Gaussian random field with the same covariance function as in Eq. (53). We use a secondorder PC expansion in terms of 4 uncorrelated random variables arising from $\mathrm{KL}$ expansion to represent the lognormal random field. Clearly, this is computationally more demanding compared to the earlier case involving a Gaussian uncertainty model since we now have to deal with a greater number of terms in the final expression for the basis vectors.

Figs. 5 and 6 show the trends of the percentage errors in the mean and the standard deviation, respectively. The effect of increasing the number of basis vectors and $\sigma$ on the accuracy is

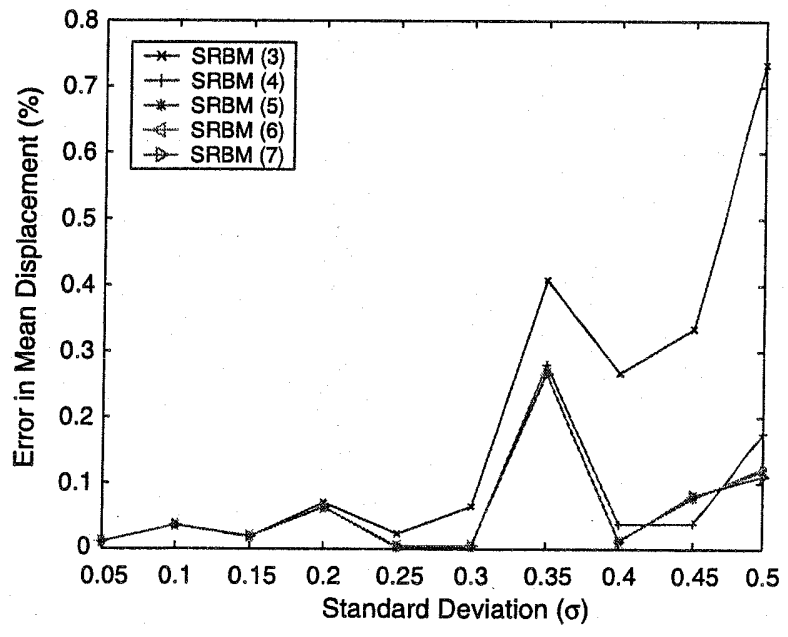

Fig. 5. Percentage error in the mean displacement at point ' $E$ ' using up to 7 basis vectors as a function of the standard deviation of the random Youngs modulus (lognormal model).

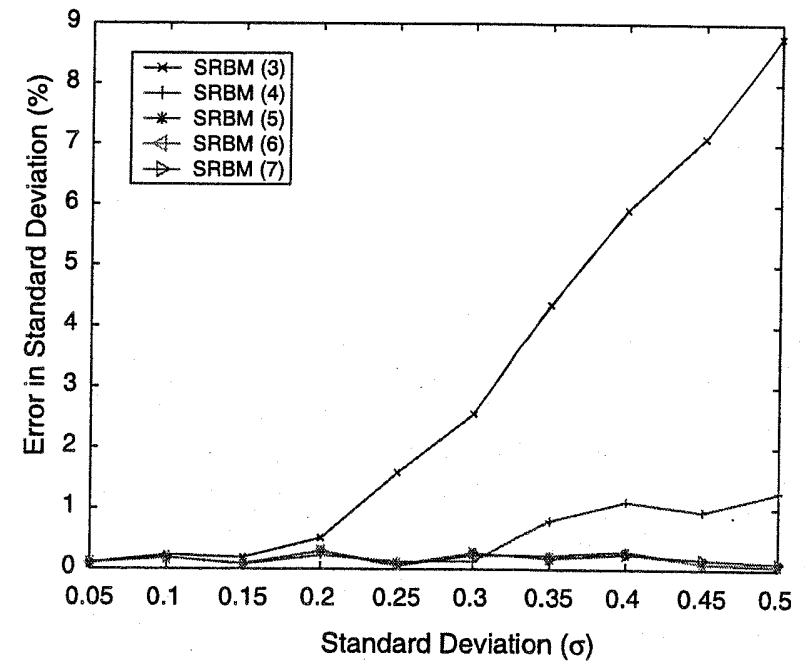

Fig. 6. Percentage error in the standard deviation of displacement at point ' $E$ ' using up to 7 basis vectors as a function of the standard deviation of the random Youngs modulus (lognormal model).

shown in these figures. It can be seen that the errors are minimal at lower values of $\sigma$. For $\sigma>0.30$ the errors in mean displacement computed SRBM(2) is higher. However, when the number of basis vectors is more than two, the error in mean displacement is always less than $0.3 \%$.

Similar trends can be observed for the percentage error in the standard deviation of displacement shown in Fig. 6. Specifically, highly accurate results can be obtained when more than two basis vectors are used - the errors are close to zero across all values of $\sigma$ up to 0.5 . Fig. 7 shows the trends of the $L_{2}$ norm of the residual error as a function of $\sigma$. From a theoretical viewpoint, the Galerkin projection scheme used here minimizes a energy norm or the $\mathbf{K}$-norm of the error, i.e. $\left\langle\{\mathbf{u}-\hat{\mathbf{u}}\}^{\mathbf{T}}\right.$ $\mathbf{K}(\boldsymbol{\theta})\{\mathbf{u}-\hat{\mathbf{u}}\}\rangle[27,28]$. Hence, the decrease in the residual error may not be strictly monotonic when the number of basis vectors is increased. This also explains why the percentage

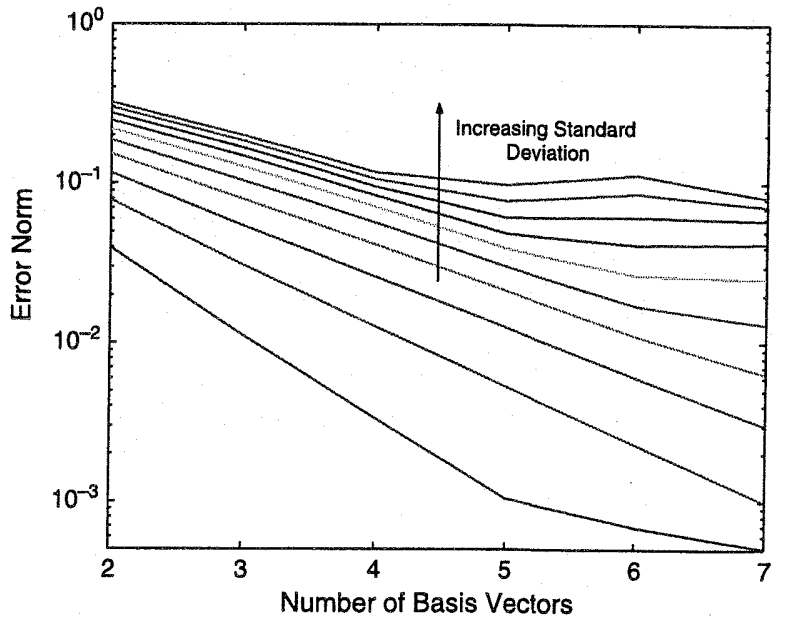

Fig. 7. Convergence of the $L_{2}$ norm of the residual error when the number of basis vectors is increased (lognormal model). 
Table 1

Comparison of the percentage error in standard deviation of displacement computed using SRBMs and the SSFEM for Gaussian model with $\sigma=0.15$ and 0.20

\begin{tabular}{llllrr}
\hline \multirow{2}{*}{ Order } & $\sigma=0.15$ & & & $\sigma=0.20$ & \\
\cline { 2 - 3 } & SRBM & SSFEM & & SRBM & SSFEM \\
\hline 1 & 5.363 & 5.374 & 10.438 & 10.455 \\
2 & 0.454 & 0.513 & 1.878 & 1.984 \\
3 & 0.076 & 0.084 & 0.531 & 0.559 \\
4 & 0.039 & 0.039 & 0.254 & 0.259 \\
5 & 0.034 & 0.034 & 0.178 & 0.179 \\
6 & 0.033 & 0.033 & 0.152 & 0.153
\end{tabular}

error in mean and standard deviation does not decrease in a monotonic sense when the number of basis vectors is increased.

Table 1 compares the percentage error in standard deviation of displacement computed using the hybrid formulation with the SSFEM of Ghanem and Spanos [10] as the order of approximation is increased. A consistent comparison is made possible since both schemes ultimately represent the solution process in terms of a PC expansion (see Section 4.2) -the term order is used here to refer to the order of the approximation in Eqs. (36) and (40). It can be noted from Table 1 that the accuracy of the hybrid projection scheme is comparable to the SSFEM. The results presented in the table are for Gaussian models with $\sigma=0.15$ and 0.20 .

It is also of interest to note that the hybrid scheme is computationally more efficient than the SSFEM. Fig. 8 shows how the hybrid schemes compare with standard PC projection schemes in terms of computational efficiency. Clearly, the reduced basis schemes are roughly 100 times faster than the SSFEM. The plot shown in Fig. 8 was obtained for the case when discretization of the plate problem results in $242 \mathrm{DOF}$. It is worth noting here that a direct solver was used to generate results using the SSFEM; the application of iterative methods is expected to lead to improvements in computational efficiency. Even so, for systems with large number of DOF, the stochastic reduced basis projection schemes proposed here will be

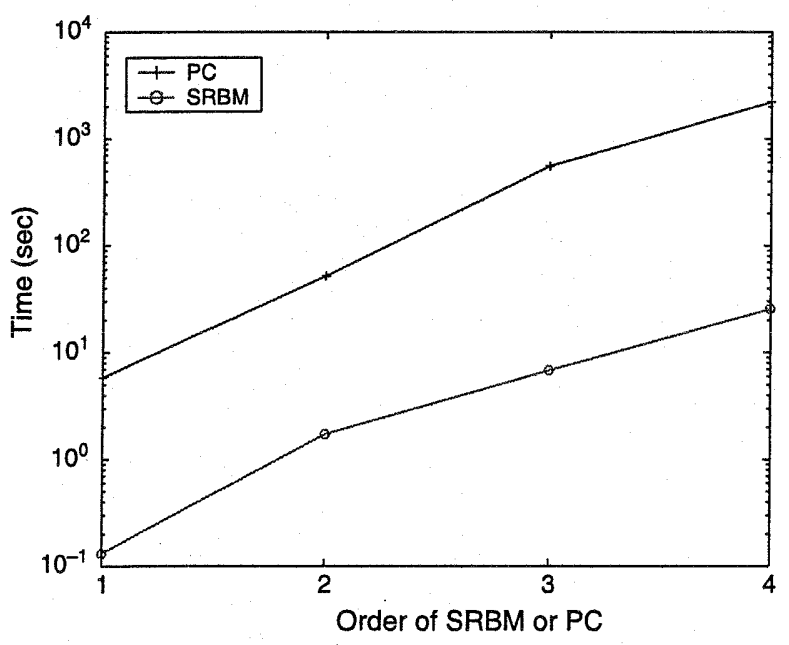

Fig. 8. Comparison of wall time taken by the SRBMs and the SSFEM of different orders to solve the plate problem with 242 DOF (Gaussian model). significantly more efficient than the SSFEM since the size of the final system of reduced-order equations is independent of the number of DOF and the number of random variables. In contrast, the size of the system of equations to be solved for the undetermined coefficients in the SSFEM is a function of the total number of DOF as well as the number of random variables, i.e. a multiple of the original number of DOF; see Section 4.2

\section{Concluding remarks}

In this paper we introduced a hybrid formulation combining stochastic reduced basis projection schemes with PC expansions for solving linear random algebraic systems of equations arising from discretization of stochastic partial differential equations. The objective of the present work was to extend SRBMs to non-Gaussian uncertainty models. To achieve this generalization, the basis vectors spanning the stochastic Krylov subspace are reformulated in terms of $\mathrm{PC}$ expansions in multidimensional Hermite polynomials. By taking advantage of the recursive representation of the stochastic basis vectors we used a pseudo-spectral decomposition technique to evaluate higher order basis vectors. As a result, a highly tractable and computationally efficient representation of stochastic basis vectors was obtained. In principle, an arbitrary number of basis vectors can be used depending on the accuracy required in the solution process. A reduced order system of equations is finally obtained, which can be readily post-processed to compute the statistics of interests using the standard properties of PC expansions.

We present detailed numerical studies to demonstrate the utility of higher order SRBMs when applied to solve a system of equations obtained by discretizing a model linear stochastic PDE in structural mechanics. We have tested SRBMs for both Gaussian and lognormal models. All the results generated are benchmarked against Monte Carlo simulation outputs. Numerical studies, as expected, suggest that higher order SRBMs give better results compared to lower order ones. Trends for the $L_{2}$ norm of the error show that even at higher standard deviations the errors decrease rapidly when the number of basis vectors is increased. Comparisons are also made with the SSFEM [10] to show that the proposed hybrid formulation gives results of comparable accuracy at a significantly lower computational cost.

\section{Acknowledgements}

This work was supported by a grant from the Faculty of Engineering, Science and Mathematics at the University of Southampton.

\section{References}

[1] Shinozuka M, Jan CM. Digital simulation of random processes and its applications. J Sound Vibr 1972;25.

[2] Hurtado JE, Barbat AH. Monte Carlo techniques in computational stochastic mechanics. Arch Comput Methods Engrg 1998;5:3-29. 
[3] Rubinstein RY. Simulation and the Monte Carlo method. New York: Wiley; 1981.

[4] Kleiber M, Hien TD. The stochastic finite element method: basic perturbation technique and computer implementation. New York: Wiley; 1992.

[5] Schuëller GI. Special issue-a state-of-the-art report on computational stochastic mechanics. Probab Eng Mech 1997;19:197-321.

[6] Yamazaki F, Shinozuka M, Dasgupta G. Neumann expansion for stochastic finite element analysis. J Eng Mech 1988;114:1335-55.

[7] Hien TD, Kleiber M. Stochastic finite element modelling in linear transient heat transfer. Comput Meth Appl Mech Eng 1997;144(111):24.

[8] Kamiński M. Stochastic second-order perturbation approach to the stressbased finite element method. Int J Solids Struct 2000;38:3831-52.

[9] Haldar A, Mahadevan S. Reliability assessment using stochastic finite element analysis. New York: Wiley; 2000.

[10] Ghanem R, Spanos P. Stochastic finite elements: a spectral approach. Berlin: Springer; 1991.

[11] Ghanem R, Spanos PA. Stochastic Galerkin expansion for nonlinear random vibration analysis. Probab Eng Mech 1993;8:255-64.

[12] Ghanem R, Brzkala V. Stochastic finite element analysis of randomly layered media. J Eng Mech 1996;122:361-9.

[13] Ghanem R. Probabilistic characterization of transport in heterogeneous media. Comput Meth Appl Mech Eng 1998;158:199-220.

[14] Anders M, Hori M. Stochastic finite element method for elasto-plastic body. Int J Numer Meth Eng 1999;46:1897-916.

[15] Maitre OL, Knio O, Najm H, Ghanem R. A stochastic projection method for fluid flow: basic formulation. J Comput Phys 2001;173:481-511.

[16] Sarkar A, Ghanem R. Mid-frequency structural dynamics with parameter uncertainty. Comput Meth Appl Mech Eng 2002;191(5499):531.

[17] Manolis GD, Karakostas CZ. A green's function method to SH-wave motion in random continuum. Eng Anal Bound Elements 2003;27: 93-100.

[18] Xiu D, Karniadakis GE. The Wiener-Askey polynomial chaos for stochastic differential equations. SIAM J Sci Comput $2002 ; 24: 619-44$.

[19] Xiu D, Karniadakis GE. Modeling uncertainty in steady-state diffusion problems via generalized polynomial chaos. Comput Meth Appl Mech Eng 2002;191:4927-48.

[20] Xiu D, Karniadakis GE. Modeling uncertainty in flow simulations via generalized polynomial chaos. J Comput Phys 2003;187:137-67.

[21] Xiu D, Karniadakis GE. Stochastic modeling of flow-structure interactions using generalized polynomial chaos. J Fluids Eng 2002;124:51-9.

[22] Xiu D, Karniadakis GE. A new stochastic approach to transient heat conduction modeling with uncertainty. Int J Heat Mass Transfer 2003;46: 4681-93.

[23] Maitre OPL, Najm HN, Ghanem RG, Knio OM. Multiresolution analysis of Wiener-type uncertainty propagation schemes. J Comput Phys 2004; 197:502-31.
[24] Maitre OPL, Knio OM, Najm HN, Ghanem RG. Uncertainty propagation using Wiener-Haar expansions. J Comput Phys 2004;197:28-57.

[25] Babuška I, Tempone R, Zouraris E. Galerkin finite element approximations of stochastic elliptic partial differential equations. SIAM J Numer Anal 2004;42:800-25.

[26] Nair PB. On the theoretical foundations of stochastic reduced basis methods. AIAA Paper 2001-1677; 2001.

[27] Nair PB, Keane AJ. Stochastic reduced basis methods. AIAA J 2002;40: 1653-64.

[28] Nair PB. Projection schemes in stochastic finite element analysis. In: Nikolaidis E, Ghiocel D, Singhal S, editors. CRC engineering design reliability handbook. Boca Raton: CRC Press; 2004 [chapter 21].

[29] Sachdeva SK, Nair PB, Keane AJ. Comparative study of projection schemes for stochastic finite element analysis. Comput Meth Appl Mech Eng. in press.

[30] Debusschere BJ, Najm HN, Pebay PP, Knio OM, Ghanem RG, Maitre OPL. Numerical challenges in the use of polynomial chaos representations for stochastic processes. SIAM J Sci Comput 2004;26: 698-719.

[31] Mathelin L, Hussaini MY. A stochastic collocation algorithm for uncertainty analysis. NASA/CR-2003-212153; February 2003

[32] Loeve M. Probability theory. 4th ed. Berlin: Springer; 1977.

[33] Wiener N. The homogeneous chaos. Am J Math 1938;60:897-936.

[34] Wiener N. Nonlinear problems in random theory. Cambridge, MA: MIT Press; 1958.

[35] Gibson LJ, Ashby MF. Cellular solids: structure and properties. 2nd ed. Cambridge, UK: Cambridge University Press; 1997.

[36] Li L, Tchelepi HA, Zhang D. Perturbation-based moment equation approach for flow in heterogeneous porous media: applicability range and analysis of high-order terms. J Comput Phys 2003;188:296-317.

[37] Vanmarcke E. Random fields: analysis and synthesis. London, England: MIT Press; 1988.

[38] Huyse L, Maes MA. Random field modeling of elastic properties using homogenization. J Eng Mech 2001;127:27-36.

[39] Sudret B, Kiureghian AD. Stochastic finite elements and reliability: a state-of-the-art report. Technical report no. UCB/SEMM-2000/08. University of California, Berkeley. p. 173.

[40] Li CC, Kiureghian AD. Optimal discretization of random fields. J Eng Mech ASCE 1993;119:1136-54.

[41] Huang SP, Quek ST, Phoon KK. Convergence study of the truncated Karhunen-Love,éve expansion for simulation of stochastic processes. Int J Numer Meth Eng 2001;52:1029-43.

[42] Zienkiewicz OC, Taylor RL. Finite element method, vol. 1. London: Butterworth Heinemann; 2000.

[43] Ghanem R. The nonlinear Gaussian spectrum of log-normal stochastic processes and variables. J Appl Mech 1999;66:964-73.

[44] Izenman AJ. Recent developments in nonparametric density estimation. J Am Stat Assoc 1991;86:205-24. 
